\title{
Aus der medizinischen Vergangenheit in Langenthal
}

\author{
Von Marta Meyer-Salzmann
}

Der Stand medizinischer Berufe im 18. Jahrhundert wird hier im bernischen Dorf Langenthal veranschaulicht. Langenthals reiche Vergangenheit wurde vor allem durch zwei Geschlechter geprägt, die Dennler und die Mumenthaler, die neben anderen Berufen und Ämtern auch als Apotheker und Wundärzte hervorragten.

Die Dennler fallen durch eine gute Ausbildung in der Apothekerkunst auf und durch einen Wundarzt, der mit seinen satirischen Veröffentlichungen an Swift erinnert. An der Schwelle des 18. Jahrhunderts lebte Joseph Dennler als Wasserbrenner. Die Wasserbrenner destillierten oft nicht nur Alkohol, sondern auch Kräuter und andere Materialien zu Heilmitteln. Friedrich Dennler-Gugelmann (1704-1758) aus einer nächsten Generation eröffnete, wie man aus den Manualen des Sanitätsrates erfährt, 1728 eine erste Apotheke in Langenthal. Er hatte seine Kunst von 1721 bis 1725 bei Andreas Grimm in Burgdorf erlernt und sich in Apotheken in Heidelberg, Kirchheim, Ladenburg und Wetzlar weitergebildet. Sein Sohn, Friedrich Dennler-Reinhard (1738-1814), der später die Apotheke übernahm, mußte sich gegen drei Mumenthaler als Heilmittelhersteller wehren, die ohne besondere Ausbildung im Gewohnheitsrecht von Vorfahren auf diesem Gebiet tätig waren. Dennler wies beim bernischen Sanitätsrat darauf hin, er habe sich nach der Lehrzeit bei seinem Vater in Basel beim berühmten Laboranten Franz Bernoulli in der Kenntnis der Drogen und in chemischen Arbeiten ausbilden lassen und dann in Frankfurt, Gießen und Düsseldorf gedient. Der Sanitätsrat bewilligte ihm allein die unbeschränkte Ausübung seines Berufes. Den drei Mumenthalern wurden nur «Simplicia» gleich den Materialisten (Drogisten) und Spezierern zugestanden, doch außerdem die Herstellung und der Verkauf von Arquebusade. Dieses war ein bekanntes Destillat aus verschiedenen Kräutern gegen Schußwunden wie auch Verletzungen anderer Art. An einer begrenzten Heilmittelbereitung hielten die Ärzte Mumenthaler durch alle Generationen fest. Die Apotheke wurde wieder von einem Nachkommen weitergeführt, von Friedrich Dennler-Rüegger (1796-1841). Er verlegte sie in ein neues Haus. Dort, an der Marktgasse 27, befindet sie sich unter dem Namen «Dennlersche Apotheke» noch heute.

Gesnerus 1/2 (1983) 
Bei den Wundärzten im Geschlecht der Dennler nimmt Andreas DennlerHellmüller (1755-1819) eine Sonderstellung ein. 1785 wurde er vom Sanitätsrat als Landarzt patentiert. Er hatte die Heilkunst offenbar, wie es damals üblich war, als ärztliches Handwerk erlernt, denn er war Feldarzt im Dragonerregiment des Erzherzogs von Toskana in Wien gewesen und hatte in Militärspitälern gearbeitet. Sein Patent bezeugt, seit langer Zeit habe niemand die Fragen so scharfsinnig und geschickt beantwortet und eine solche Kenntnis der medizinischen Theorie an den Tag gelegt wie er. Diesem jungen, hoffnungsvollen Mann werde die Bewilligung zur Ausübung der Arzneikunst auf dem Land gerne erteilt.

Als praktizierender Arzt in Langenthal war er geschätzt; als Freidenker und satirischer Schriftsteller erregte er Anstoß. Die Französische Revolution und ihre Auswirkungen wühlten ihn auf. Er sah viel Unrecht, wollte es beseitigen, erkannte die Unmöglichkeit und verfiel in beißenden Spott. Der Menschenkenner wußte, wie das Leben besser und schöner sein sollte, und wie es sich grausam und bitter zeigte. Seine Schreibweise ist weitläufig, sprunghaft und manchmal derb.

Das erste Buch trägt den Titel: «Die ganze Natur, Himmel und Hölle in einer Nuß» (Helvetien [Stäfa] 1799). Dort beschäftigt ihn die Entstehung des Menschen. Er entwickelt die Idee von einer beseelten Welt. In seiner Vorstellung entwirft er ein ausstrahlendes Feuer- oder Lichtwesen, das die tote, ruhende Natur belebe, wobei die fähigeren Lebewesen aus mehreren farbigen, die einfacheren aus weniger Strahlen gemodelt werden, und je nach dem vorgefundenen Stoff werde der Mensch geformt. Die Seele bestehe aus Billionen zarter, elastischer und unzerstörbarer Lichtfasern. Ihr Hauptsitz sei die Leber; von da verbreite sie sich durch den ganzen Körper. Der Verdauungsvorgang werde von der Seele beeinflußt; er beschreibt ihn in Einzelheiten, auch in Verbindung mit der Gallenblase und der Bauchspeicheldrüse. Dann baut er Hypothesen, wie dauernd Neues erzeugt und Überflüssiges abgestoßen werde, und doch bleibe jeder der nämliche Boissy d'Anglas oder Merlin (beide waren Revolutionspolitiker). Er zielt hin auf die Gleichgültigkeit und Selbstzufriedenheit mancher Menschen in politisch erregender Zeit. Den Ort, wo er Pfeile auf Zeitereignisse, auf Kirche und Amtsleute abschießt, nennt er «Papimanien». Einerseits sagt er, man schlafe dort 18 Stunden im Tag, andererseits mahnt er aufrüttelnd, die Erde sei unser Haus, hier müssen wir bleiben und wohnen, der Stall müsse ausgemistet, von Fanatikern, Despoten und Pfaffen gesäubert werden. Er deutet auf unser Unterbewußtsein, indem er schreibt: «Im Schlafe blättert 
die Seele im alten Testament, sie spürt in Archive», dann auf ein Weiterleben nach dem Tode, «Wir werden ernten, was wir jetzt säen», womit er ein neues Dasein auf dieser Erde meinte. Dennler ist erfüllt von der «Metempsychosis aus India» (Seelenwanderung aus Indien).

Zwei längere Beiträge, «Homilie über ein Wort des Diogenes von Synope» und «Neueste Reise von Lissabon nach Kamtschatka und dem Nordpol», erschienen 1805 und 1806 in Johann Heinrich Zschokkes Monatsschrift «Isis». Zschokke bezeichnet ihn im Vorwort 1805 als einen unbekannten Mann in einem schweizerischen Dorf, der mit Begierde lese, was er an Schriften auftreiben könne. Die Ausführungen nennt er eine Philippika gegen menschliche Torheiten. Der erste Beitrag geht von Diogenes aus, der am hellichten Tag mit der Laterne vernünftige Menschen gesucht habe. Dennler schweift in weite Vergangenheit und sieht viel Unvernunft. Doch er schätzt Baco von Verulam (1561-1620), der in neuerer Zeit als erster gewagt habe, vernünftig zu denken und öffentlich dazu zu stehen. Auch Kopernikus begeisterte ihn, «dieser geweihte Dolmetsch der wundervollen Natur». Die Erde «im Mittelpunkt eines vielgestirnten kristallenen Gewölbes war von jeher der berüchtigte Tummelplatz aller Thorheit ... Heut aber, wenn man bey sternheller Nacht den Kopf durchs Fenster stekt, um nach Wind und Wetter zu schauen, ist dieser kleine Anlaß für den auch nur Halbdenkenden trostvoller ... Der Geist der Menschen, indem sein Blick auf die flammenden Irrsterne und Sonnen des Universums fällt, erblickt dann nicht mehr die koboldsartigen Götter der Vorwelt in ihnen, sondern liest nur den goldnen Anfangsbuchstaben des ewigen Seyns, wovon der bleiche Schimmer der Milchstraße und der Nebelsterne den zweiten Buchstaben ahnen läßt.»

Im zweiten Beitrag unternimmt er eine Phantasiereise, ähnlich wie Swifts Gulliver. Swift kleidete seine Kritik an Staat, Gesellschaft und Moral Englands in abenteuerliche Märchen. Dennler jedoch geht in Mitteleuropa gradlinig zum Angriff über. Im Cis-Rigaischen befänden sich Künste und Wissenschaft auf dem höchsten Gipfel. Man dürfe jetzt alles wissen, reden, schreiben, denken, drucken, ohne Ausnahme, nur eine Kleinigkeit - die Wahrheit nicht. Die Trans-Riganer seien noch größer: Man bezahle und schicke die allergelehrtesten Leute in die warmen Urstammländer wie Rom, Karthago, Memphis, Delphi, Phönizia, Babylon und Ninive, lasse alle verschütteten Gewölbe, Keller, Mordgruben, Kerker, Abtritte, Tempel und Boudoirs unserer Vorväter sorgfältig durchsuchen, alle noch vorhandenen Reste von Weisheit und Wissenschaft fleißig in Säcke fassen und mit großen Kosten heim ins Land der Knute in die Weisheitsmagazine bringen. 
Es beschäftigt ihn erneut die Menschwerdung: Wie eine Festung ihre Garnison einschlürfe und wieder ausspeie, pumpe alles was Atem habe, bei jedem Zug Regimenter vegetierender Seelen ein und aus; doch nur die gleichgemodelten Seelen bekleiden und reproduzieren sich in gleichgemodelten Stoffen, Haselnußseelen in Haselnußstauden, Meerwunder und Erdtölpel in ihresgleichen, womit er ausdrückte, daß alles immer wieder vorhanden sei. Und die Laus wie der Lauskerl seien vom gleichen Stoff organisiert, doch nicht durchaus gleich gebündelt.

Andreas Dennler kam wegen aufrührerischer politischer Reden ins Gefängnis und verursachte auch einen Fensterladen-Prozeß, da die mit Bildern bemalten Laden seines Hauses als Spott auf die Geistlichkeit betrachtet wurden.

Im «Bürger Quixots aus Uchtland» (London [Baden] 1817) entwirft er unter anderem ein satirisch gemeintes Programm, die Kindheit um manches Jahrzehnt zu verlängern, damit der Körper eine bewunderungswürdige Größe und Dauer, besonders aber der physische und moralische Magen eine solche Kraft erlange, daß beide mit der Zeit im Stand wären, Kieselsteine und Hufnägel, grobe und reine Wahrheit, Schimpf und Ernst zu verdauen. Er erklärt, daß Leib und Seele in ihrer Entwicklung zu stark beschleunigt werden und deshalb erlahmen. Wenn die Leute endlich in ihrem fünfzigsten oder sechzigsten Jahr von ihrer gesunden Vernunft einen Gebrauch zu machen versuchen, der nur wenig Anstrengung koste, sei ihr Leben zu Ende. Er fragt, was die Plutarche, Rousseaus, Tissots, Basedows, Campes, Salzmanns und übrigen Philanthropen und Erziehungsräte erreicht hätten.

Für die Erwachsenen in den verschiedenen Berufen legt Dennler eine entsprechende Diätetik (Lebensordnung) dar. Beherzigenswertes und Doppelsinniges mischen sich. Man ist nie ganz sicher, ob er meint: Sage mir, was du issest, wie du wohnst, und ich will dir sagen, wer du bist, ob Fuhrmann, Minister, Tanzmeister ... Und ironisch begründet er, wie wenig Bedeutung dem Gehirn für den Verstand zukomme. Schon sein Aussehen sei hiezu belanglos, und er beruft sich auf Sektionen. Er stellte einen Unterschied fest zwischen dem Gehirn eines Wirtes aus dem nahen Murgenthal, der bis zuletzt in geistig gutem Zustand war, und dem, was er in Militärspitälern beobachtete. Des Wirtes «graue Substanz» sei fester als bei anderen gewesen. «Die Seh'nerven von ihrem Ursprung an, bis wo sie in die Orbitas [sic] laufen, fast lederhart und hirsern, doch weit feiner», schreibt er. So verquickt er Konkretes mit Abstraktem in unbeschwerter eigenwilliger Art.

Andreas Dennler war sehr belesen und bewandert. Zwingli und Calvin, 
Montaigne, Pascal, Leibniz, Wolff, Voltaire, Lafontaine, Kant, Jung-Stilling, Lavater und Pestalozzi werden erwähnt, aber auch Hippokrates, Mohammed, Albrecht Dürer, Lukas Cranach, Hans Holbein und manche andere. Aus der weiten Welt schöpfte er seine Gedanken zur Humanität und setzte sich auf seine ganz persönliche Weise dafür ein.

Anders stellte Johann Georgius Mumenthaler-Geiser (1704-1780) seine Belesenheit in den Dienst der Mitwelt. Das körperliche Wohlbefinden des Einzelmenschen stand für ihn im Vordergrund. Sein Großvater war der Heilmittelhersteller Georg Mumenthaler-Dennler, sein Vater der Schnittund Wundarzt Friedrich Mumenthaler-Witschy (1677-1736), der nebenher als Zollverwalter, Postmeister und obrigkeitlicher Tuchmesser wirkte und bei seinem Ableben als gewesener Apotheker bezeichnet wurde. Johann Georgius trat als 23 jähriger bei seinem Vater in eine dreijährige Wundarztlehre ein. Nach zwei weiteren Jahren als Geselle bestand er vor der Chirurgischen Societät in Bern die Meisterprüfung. Ein 1765 von ihm ausgestellter Lehrvertrag hat sich als Rarität erhalten und gibt Aufschluß über die Vereinbarungen, die zwischen ihm und dem Vater eines Lehrlings getroffen wurden. Darin steht unter anderem: «dann verspriche ich seinem Sohn in seiner Lehrzeit alle Handgrieff in der Chirurgiae, die principiae in Medicin und Apotheker-Kunst alle nothwendige Wüssenschaft zu zeigen; auch sollen Thme alle Schriften zugestellt werden abzuschreiben, und sich darinn zu Exercieren. Summa, ich werde trachten Ihme solches auf eine Methodische Art und Weise einzugeisten.» Das Abschreiben aus des Meisters Schriften war des Lehrlings «Gewerbeschule», die er bei seinem Meister durchlief.

Die Krone seines ärztlichen Schaffens bildeten schriftstellerische Arbeiten. Zwei in Privatbesitz noch erhaltene Bände, wovon der eine den anderen fortsetzt, haben zwar Oktavformat, umfassen jedoch 702 und 956 Seiten. Die Aufschrift lautet: «Guldenes Kleinod der Medicinischen Geheimnussen» (Langenthal o. J.). Im zweiten Band gibt er 226 Werke an, auf die er sich gestützt hat. Johann Georgius zeigt sich als philosophischer Lehrer. Er sucht die Fragen von Gesundheit und Krankheit gedanklich zu lösen und nimmt dazu sein großes Wissen aus der Kulturgeschichte zu Hilfe. Immer wieder holt er weit aus; er erinnert an die ägyptische Medizin und an die Druiden als Priesterärzte; er nennt die klassischen medizinischen Autoren Hippokrates, Galenus, Celsus, Aetius und Avicenna, aber auch Naturforscher wie Aristoteles und Plinius, Denker wie Plato und Sokrates, Epiker wie Homer und Vergil, Schriftsteller wie Cicero und Plutarch, Dichter wie Horaz und Ovid. 
Aus neuerer Zeit sind ihm Ärzte bedeutsam, aus der Schweiz Paracelsus, Felix Platter, Johann Jakob Wepfer, Johann Conrad Brunner, Theodor Zwinger und der Chirurg Fabricius Hildanus, aus der Ferne Daniel Sennert, Friedrich Hoffmann und Herman Boerhaave. Er zitiert Lebensweisheiten und ärztliche Erkenntnisse in Latein, hie und da auch in Griechisch.

Wir greifen aus dem Inhalt heraus: Was man ißt und trinkt, ist für die Gesundheit entscheidend. Im Zusammenhang mit dem Brot erörtert er die Getreidesorten, Mehlsorten und die Brotbereitung. Gelobt wird das gute, reine Wasser, womit man unter anderem Kopfschmerzen, Schnupfen und böse Mandeln heilen könne, und er verweist auf Celsus. Ein Quentlein «tartarus vitriolatus» oder «arcanum duplicatum» (beides waren Bezeichnungen für schwefelsaures Kalium), in Wasser aufgelöst, sei ebenfalls von Nutzen. «Denn auf solche Weise löset es allen zähen Schleim und Galle sammt den Saltztheilen, spühlet den Magen und die Gedärme rein aus, und eröffnet die in die Milch-Adern gehenden Mündungen, damit der Nahrungssaft desto besser ohne Hinderniß in die Milchgefäße dringen könne, auch verdünnet solches das Geblüte, und machet es zu einem freien Lauffe durch die Adern geschickt.» Gutes Brot und reines Quellwasser oder Regenwasser scheinen ihm übrigens ausreichend, den menschlichen Körper in Vollkommenheit zu erhalten.

Das Fasten und die Mäßigkeit im Essen befürwortet er mit der Heiligen Schrift, mit Hippokrates, Galenus und Paracelsus. Zu einem sanften, ruhigen und gesunden Schlaf seien frische Luft, nicht zu kalte und nicht zu warme Räume notwendig. Die ungünstige Haltung während des Schlafes wird in ihren Auswirkungen erläutert. Das Atmen sei wichtig, lautes Lesen dienlich, weil dadurch das Zwerchfell zu stärkerer Bewegung angetrieben werde. Noch viel mehr helfe Singen zu einem langen und gesunden Leben, wobei er sich auf Giorgio Baglivi (1668-1707) beruft. Er warnt vor zu vielen Arzneiverordnungen und belegt dies mit Wepfer, Brunner und Montanus. Gegen langwährenden Kummer, der wie ein nagender Wurm der Gesundheit schade, empfiehlt er einen guten Wein, dem auch die Heilige Schrift die Tugend beilege, daß er das menschliche Herz erfreue (Psalm 104; Sirach, Kap.31 und 32). Bei allzu großer plötzlicher Freude nehme man zur Beruhigung Destillate von «Mayenblumen», Melisse, Lindenblüten und Orangenblüten oder ein «Marggraphisches Pulver» (nach dem Berliner Chemiker Andreas Sigismund Marggraf, 1709-1782).

Den harmonischen Gemütszustand bezeichnet er als Quelle der Gesundheit. Er umschreibt ihn so, wie es wohl seinem eigenen Wesen entsprach: 
«Auch kann zu einem ruhigen Gemüthe die Einsicht mathematischer Sachen, wie nicht weniger die Erkänntniß der Natur und Anschauung der Wunder-vollen Wercke Gottes darinnen, auch Forschung der Chimie, Historie, Sprachen und anderer schönen und herrlichen Wissenschaften mehr, gar viel beytragen: Denn alles dieses sind Stücke, welche nicht nur ihren wircklichen und unschätzbaren Nutzen haben, sondern auch wegen der Ergötzbarkeit, so darmit verknüpfet ist, billig hoch und werth zu achten, weil sie das Gemüthe vergnügen und ermuntern, als wodurch eben der Leib gesund erhalten wird.»

Eine Keramikfigur von $26 \mathrm{~cm}$ Höhe stellt ihn als leicht gebückten alten Mann mit nachdenklichem Gesicht und bäuerlicher Kleidung dar.

Seine Tatkraft trugen die Söhne Johann Georg Mumenthaler-Marti (1734-1814) und Jacob Mumenthaler-Marti (1737-1787) weiter; sie heirateten Schwestern. Jacob besuchte nach der wundärztlichen Lehre Kurse an der Universität Straßburg; darauf förderte er seine Ausbildung in Berlin. Dort bestand ein «Collegium medico-chirurgicum» mit einem Theatrum anatomicum. Bevor sich Jakob in Langenthal niederließ, trat er für zwei Jahre in holländischen Kriegsdienst. Auf seiner letzten Reise gelangte er von Rotterdam über Gibraltar, Malaga und Tunis nach Smyrna. Nach der Rückkehr diente er im Schweizerregiment von Diesbach, das sich im Siebenjährigen Krieg auszeichnete. Er blieb vier Jahre; die Entlassungsurkunde bescheinigt, daß er als Chirurg geachtet war. Später wurde er Oberleutnant im ersten oberaargauischen Regiment. Ein Ölbild zeigt ihn in jener Uniform; er weist auf eine Europakarte.

Bevor wir auf seinen Bruder Johann Georg eingehen, wenden wir uns seinem Sohn und seinem Enkel zu. Der Sohn Johann Georg MumenthalerZulauf (1769-1825) betrat ebenfalls die wundärztliche Laufbahn und besuchte die Universität Straßburg. Er ging zur Weiterbildung in die Pariser Spitäler «Hôtel-Dieu» und «Hôtel des Invalides». Als Arzt in Langenthal vertrieb er unter anderem «Mumenthaler Kräuter-Thee» sowie «HauptFlußpulver» gegen schwaches Gedächtnis, schwache Sehkraft und Schwindel. Auf einem Ölbild trägt er die bernische Infanterie-Offiziersuniform.

Sein Sohn wurde der letzte Vertreter der Ärztedynastie. Johann Friedrich Mumenthaler-Gugelmann (1805-1852) war nicht mehr Handwerksarzt, denn er studierte an der 1805 gegründeten Akademie in Bern und dann an der Universität Heidelberg. Eingebundene Kolleghefte aus Bern sind erhalten. Laut seinem Arztpatent durfte er die Medizin und Chirurgie mit Einschluß der Geburtshilfe ausüben, eine Hausapotheke halten und von 
anderen patentierten Medizinalpersonen verschriebene Verordnungen zubereiten und dispensieren. Sein Wohnhaus, das vier oder fünf Generationen der Ärzte Mumenthaler beherbergt hat, steht heute noch an der Marktgasse 32. Johann Friedrich war der Hüter eines aufschlußreichen Nachlasses, der zum Teil im Ortsmuseum Langenthal und bei Privaten aufbewahrt wird.

Wir kehren zurück zu Johann Georg Mumenthaler-Marti. Sein Studienaufenthalt in Straßburg 1753/54 ist dokumentiert. In seinen nachgeschriebenen Vorlesungen in Pathologie lassen sich 354 dicht beschriebene Seiten zählen. Es wird über 67 Krankheitsgruppen berichtet. In erster Linie wurden in Straßburg theoretische Grundlagen über pathologische Vorgänge vermittelt, Symptome erklärt, Krankheitsursachen dargelegt und Prognosen gestellt, bevor Behandlungsvorschriften und Medikamente angegeben wurden. Die Rezepte enthalten viele Bestandteile. Man versuchte, Altbewährtes mit Neuem vorsichtig zu verbinden. Wir treffen in der gleichen Anweisung pflanzliche, tierische, mineralische und chemische Zutaten. Der lateinische Fachwörterschatz ist umfangreich.

Er brachte aus Straßburg drei Atteste heim. Zwei davon über besuchte chirurgische Kurse waren aufzuspüren; der dritte über Pathologie fehlt uns. Professor Georg Heinrich Eisenmann (1693-1768) bestätigt ihm, daß er einen «Cursum Demonstrationum Anatomicarum et Operationum Chirurgicarum frequentiret und, in denen Morgenstunden denen Dissectionibus Cadaverum mit rühmlichem Fleiß, Auffmercksamkeit, wie auch Bescheidenheit beygewohnet». Im Attest von Professor Philipp Heinrich Böckler (1718-1759) steht, daß Johann Georg Mumenthaler «das Collegium Chirurgicum über Plattners Einleitung in die Wundarzneykunst mit besondern und rühmlichen Fleiß und Auffmercksamkeit allezeit besuchet, anbey in geziemender Bescheidenheit niemahlen etwas ermangeln lassen». Ähnlich wie in handwerklichen Lehrbriefen sind auch an der Universität Fleiß und Charaktereigenschaften beachtet worden.

Das Buch mit den dargestellten Vorlesungen zur inneren Medizin, verfaßt von einem dem ärztlichen Handwerksstand angehörenden 20 jährigen Mann, ist beeindruckend. Er bestand 1761 die Prüfung bei der Meisterschaft der Schnitt- und Wundarzneikunst in Burgdorf.

Der Oberaargau brachte weitere tüchtige Wundärzte hervor. Daß sich in Langenthal ohne Unterbruch sechs Generationen Mumenthaler mit Ansehen der Heilkunst widmeten, ist eine besonders erfreuliche Feststellung. Im Ortsmuseum Langenthal ist ein Raum der Hinterlassenschaft den Mumenthaler-Ärzten mit einigen Hinterlassenschaften gewidmet. 
PS. Eine ausführlichere Arbeit über Langenthals Handwerksärzte und Apotheker mit entsprechendem Quellenverzeichnis erscheint 1984 als Sonderausgabe der «I angenthaler Heimatblätter». Diese werden herausgegeben von der «Stiftung zur Förderung wissenschaftlich-heimatkundlicher Forschung über Dorf und Gemeinde Langenthal». Die hauptsächlich benutzten Quellen, die auch für diesen Beitrag gelten, seien hier vermerkt. Für die Dennler: Manuale des Sanitätsrates im Staatsarchiv des Kantons Bern, Manuale der Chirurgischen Societät in der Burgerbibliothek Bern, Gemeindemanuale und Kirchenbücher im Burgerarchiv Langenthal, Literatur von Andreas Dennler in der Zentralbibliothek Zürich. Für die Mumenthaler: Manuale des Sanitätsrates und der Chirurgischen Societät wie oben, das Straßburger Vorlesungsbuch und andere Handschriften und Druckschriften, eine Kopie des Lehrvertrags, Bücher aus dem Nachlaß der Ärzte Mumenthaler, Apothekerschränke und Arzt-Utensilien im Ortsmuseum Langenthal (zur Zeit wegen Restauration geschlossen). Aus Privatbesitz standen zur Verfügung: Lehrbriefe, Meisterbriefe, Lehrvertrag (Original), Atteste der Universität Straßburg, Arztpatente, eine kleine Familienchronik mit Stammtafel, Literatur von Johann Georgius Mumenthaler, die in Bibliotheken nicht aufzufinden war, Briefe und Notizen sowie Gemälde und Keramik. Verschiedenes wird später in Kopien dem Ortsmuseum Langenthal zugestellt werden.

\section{Summary}

This article deals with the professional training of apothecaries and surgeons in an $18^{\text {th }}$ century village in the Canton of Berne. During several generations, the members of two families displayed a high standard of professional education. Two apothecaries worked in shops or laboratories in Germany. Some of the surgeons passed an apprenticeship and studied subsequently at the University of Strassburg. One of them took notes of lectures on pathology, methods of cure and medical prescriptions. A surgeon wrote two books about health-care and another published different works as a satirist. Both knew many classical writers and refered to them.

Dr.h.c. Marta Meyer-Salzmann

Ackersteinstraße 178

CH-8049 Zürich 